\title{
Optical Coherence Tomography Biomarkers Predicting Visual Acuity Change after Intravitreal Bevacizumab Injections for Macular Edema Secondary to Branch Retinal Vein Occlusion
}

\author{
Ori Segala, b Roy Yavnieli ${ }^{a}$ Michael Mimounic Gilad Rabinab, d Noa Geffen ${ }^{b}$ e \\ Elad Moisseiev ${ }^{a, b}$ Arie Y. Nemet ${ }^{a, b}$

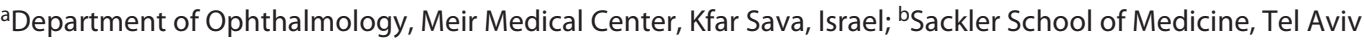 \\ University, Tel Aviv-Yafo, Israel; 'Department of Ophthalmology, Rambam Health Care Campus and the \\ Technion-Israel Institute of Technology, Haifa, Israel; ${ }^{\mathrm{d} D e p a r t m e n t}$ of Ophthalmology, Tel Aviv Sourasky Medical \\ Center, Tel Aviv-Yafo, Israel; 'Department of Ophthalmology, Rabin Medical Center, Petah Tikva, Israel
}

\section{Keywords}

Branch retinal vein occlusion - Bevacizumab - Optical coherence tomography $\cdot$ Response $\cdot$ Macular edema

\begin{abstract}
Purpose: This study aimed to identify baseline optical coherence tomography (OCT) factors in branch retinal vein occlusion (BRVO) that predict response to bevacizumab injections. Methods: It is a retrospective case series of consecutive patients that underwent OCT at diagnosis, and the central macular thickness (CMT), extent of disorganized retinal inner layers, and outer retinal layers including external limiting membrane, ellipsoid zone (EZ), and cone outer segment tips (COST) were measured. Patients received 3 consecutive monthly injections of bevacizumab followed by pro re nata treatment. The main outcome measure was improvement in best-corrected visual acuity (BCVA) after 1 year. Results are expressed as mean $\pm \mathrm{SD}$, and $p$ value $<0.05$ was considered statistically significant. Results: Overall, 66 eyes of 66 patients, with an average age of $68.5 \pm 11.4$ years, were included. The mean BCVA improved significantly from 0.68 logMAR at baseline to $0.50 \log M A R$ at 1 year $(p<0.001)$. Baseline logMAR BCVA $(r=0.41, p<0.001)$ and CMT $(r=0.23, p=$
\end{abstract}

karger@karger.com www.karger.com/oph

Karger $\stackrel{\text { ' }}{5}$

GOPEN ACCESS
C 2021 The Author(s).

Published by S. Karger AG, Basel

This is an Open Access article licensed under the Creative Commons Attribution-NonCommercial-4.0 International License (CC BY-NC) (http://www.karger.com/Services/OpenAccessLicense), applicable to the online version of the article only. Usage and distribution for commercial purposes requires written permission.
$0.04)$ were associated with improvement while EZ ( $r=-0.24$, $p=0.05)$ and COST $(r=-0.32, p=0.01)$ disruption with deterioration in BCVA. Conclusion: In patients with naïve BRVO treated with bevacizumab, BCVA improvement at 1 year can be predicted from baseline BCVA, CMT, extent of COST disruption, and $E Z$ disruption.

(c) 2021 The Author(s).

Published by S. Karger AG, Basel

\section{Introduction}

Retinal vein occlusion (RVO) is one of the most common retinal vascular diseases, and macular edema (ME) is the most common cause of visual impairment in people with these diseases [1]. RVO systemic risk factors include older age, hypertension, arteriosclerosis, diabetes mellitus, hyperlipidemia, vascular cerebral stroke, blood hyperviscosity, and thrombophilia and ophthalmic risk factors include glaucoma, decreased ocular perfusion pressure, retinal arteriolar narrowing, and arteriovenous nicking [2]. To date, various treatments have been reported to be effective in reducing $\mathrm{ME}$ associated with BRVO, such as intravitreal injections of anti-vascular endothelial growth factor [3-7], intravitreal injections of triamcinolone ace- 
tonide [8], dexamethasone intravitreal implant [9], grid laser photocoagulation [10], and pars plana vitrectomy combined with internal limiting membrane peeling [11]. However, not all patients benefit from the treatment, and sometimes visual acuity (VA) does not improve even if there is significant improvement in ME itself, suggesting that there may be several factors that predict the final visual outcome independent of the agent used to treat ME [12].

Several studies have been conducted in order to identify predictive factors for treatment success. Baseline VA, patient age, presence of macular ischemia, and response to first injection are regarded as predictive factors of final VA in patients with ME due to BRVO [13-15]. The purpose of the present study was to predict bevacizumab injection response for BRVO patients by identifying baseline OCT factors.

\section{Methods}

The study adhered to the tenets of the Declaration of Helsinki and was approved by the Institutional Review Board (IRB) of the Meir Medical Center, Israel.

\section{Study Population}

It is a retrospective case series of consecutive naïve BRVO patients that were treated with bevacizumab injections between January 2012 and March 2019 at the Meir Medical Center, Israel. Patients diagnosed with naïve BRVO and treated with 3 initial consecutive monthly intravitreal injections of bevacizumab followed by administration pro re nata and with a follow-up of at least 12 months were included in this current study. Patients with myopia of $>5 \mathrm{D}$ and any intraocular surgery $<3$ months prior to study enrollment or during the follow-up period were excluded from this study. Patients with comorbidities that can affect the study results, such as diabetic macular edema, vitreomacular traction, or uveitis, were excluded. All follow-up visits included VA exam, complete slit-lamp ocular examination, spectral-domain (SD)-OCT scans, and on the first visit or when needed fluorescein angiography. After the patients received 3 consecutive monthly intravitreal injections of bevacizumab, they were followed by pro re nata protocol.

\section{Data Collection}

The medical files of the included patients were reviewed, and patient data, including demographics, dates of all visits and injections, best-corrected visual acuity (BCVA), and OCT findings, were collected. BCVA was converted to logMAR for statistical analyses but was recorded in Snellen.

\section{Optical Coherence Tomography}

SD-OCT (Spectralis OCT; Heidelberg Engineering, Heidelberg, Germany) testing was performed through a dilated pupil, centered on the fovea and optic nerve head, using the established "posterior pole" protocol that provides high-speed scans with a dimension of $30 \times 25$ and a $120-\mathrm{mm} \mathrm{B}$-scan spacing [16]. Each
OCT scan was evaluated by 2 masked graders, and if there was disagreement, a third grader evaluated the scans in order to decide. All OCT images were carefully reviewed to exclude patients with insufficient data and poor-quality images, including inability to observe properly the retinal layers due to retinal hemorrhages.

\section{Image Analysis}

Images were analyzed as per the procedures performed by Sun et al. [17]. Seven OCT scans were used, including the central foveal scan, 3 immediate scans above, and 3 immediate scans below the central foveal scan. Central macular thickness (CMT) and retinal volume in the central $6 \mathrm{~mm}$ were recorded from Spectralis OCT after segmentation and manual error corrections. The presence of disorganized retinal inner layer (DRIL), epiretinal membrane, intraretinal cysts, small hyperreflective foci, large irregularly shaped hyperreflective foci, subretinal fluid (SRF), cone outer segment tip (COST) disruption defined as a highly reflective line disruption observed between the ellipsoid zone (EZ) and retinal pigment epithelium [18], external limiting membrane (ELM) disruption, and EZ disruption was evaluated in the 1-mm circle, centered on the fovea. The vitreomacular interface, which included findings such as partial posterior vitreous detachment or vitreomacular adhesion, was documented. The amount of hyperreflective foci was classified as few (0-10), moderate (11-20), or many (above 20), and the length of COST disruption was measured as the length of the hyporeflective line above the retinal pigment epithelium.

\section{Bevacizumab Injection Procedure}

After draping the patient and using a lid speculum, povidone iodine $4 \%$ was placed on the conjunctiva, followed by a 30 -gauge needle intravitreal injection of bevacizumab $(1.25 \mathrm{mg} / 0.05 \mathrm{~mL})$.

\section{Statistical Analysis}

All data collected were inserted into a Microsoft Excel 2007 (Microsoft Corporation) spreadsheet. Statistical analyses were performed using Minitab Software, version 17 (Minitab Inc., State College, PA, USA). Results are expressed as mean \pm SD, median (range), or $N(\%)$. Pearson correlation was used to analyze the relationship between baseline demographics and OCT measurements and delta BCVA. For comparison of continuous variables before and after treatment, the paired $t$ test was used. The Student $t$ test was used for comparison between 2 group averages, and oneway analysis of variance was used for comparison of $>2$ group averages. We then performed partial least squares multiple regression in order to determine the baseline variables that enabled to predict delta BCVA. $p$ value $<0.05$ was considered statistically significant.

\section{Results}

A total of 93 eyes of 93 patients were diagnosed with BRVO during the follow-up period. Of them, 27 patients were excluded from this study - 13 patients with poorquality OCT images, 8 patients with macular comorbidities such as diabetic macular edema or vitreomacular traction, 5 patients underwent intraocular surgery $<3$ 
Table 1. Correlation between baseline demographic and OCT measurements and delta BCVA for BRVO patients

\begin{tabular}{llll}
\hline & \multicolumn{2}{l}{ Delta BCVA } & \\
\cline { 2 - 4 } & Pearson correlation & point estimate $(95 \% \mathrm{Cl})$ & $p$ value* \\
\hline Baseline logMAR & 0.41 & $0.32(0.15,0.48)$ & $<0.001$ \\
Age, years & -0.03 & $-0.001(-0.01,0.01)$ & 0.82 \\
CMT, per $100 \mu \mathrm{m}$ & 0.23 & $0.05(0.00,0.10)$ & 0.04 \\
Volume, per $1 \mathrm{~mm}^{3}$ & 0.11 & $0.05(-0.003,0.1)$ & 0.36 \\
DRIL, per $100 \mu \mathrm{m}$ & 0.07 & $0.008(-0.02,0.04)$ & 0.59 \\
Small intraretinal cysts & -0.03 & $-0.01(-0.09,0.07)$ & 0.79 \\
Medium intraretinal cysts & 0.01 & $0.005(-0.11,0.12)$ & 0.93 \\
Large intraretinal cysts & -0.14 & $-0.13(-0.36,0.10)$ & 0.25 \\
ELM disruption, per $100 \mu \mathrm{m}$ & 0.03 & $0.02(0.003,0.05)$ & 0.79 \\
EZ disruption, per $100 \mu \mathrm{m}$ & -0.24 & $-0.03(-0.06,0.00)$ & 0.05 \\
COST disruption, per $100 \mu \mathrm{m}$ & -0.32 & $-0.06(-0.09,-0.01)$ & 0.01 \\
SRF width, per $100 \mu \mathrm{m}$ & 0.05 & $0.005(-0.02,0.03)$ & 0.69 \\
SRF height, $\mu \mathrm{m}$ & 0.06 & $0.34(-1.05,1.72)$ & 0.63 \\
\hline
\end{tabular}

BCVA, best-corrected visual acuity; OCT, optical coherence tomography; BRVO, branch retinal vein occlusion; SRF, subretinal fluid; EZ, ellipsoid zone; COST, cone outer segment tip; CMT, central macular thickness; DRIL, disorganized retinal inner layers; ELM, external limiting membrane. * Pearson correlation was used to analyze the relationship between baseline demographics, OCT measurements, and delta BCVA.

months prior to study enrollment, and 1 patient with myopia of $>5$ D. Sixty-six eyes of 66 patients, of which $37.9 \%$ were of male gender, met the inclusion criteria and were included in this study. The average age of the patients was $68.5 \pm 11.4$ years. The mean BCVA improved significantly from $0.68 \log$ MAR at baseline to $0.50 \log$ MAR at 1 year $(p<0.001$, paired $t$ test). Patients received an average of $7.6+/-2.1$ intravitreal bevacizumab injections by 1 year, and none received laser treatment. CMT improved significantly from 552.5 to 398.6 micron $(p<0.001$, paired $t$ test).

Baseline BCVA (Pearson $r=0.41, p<0.001$ ) and CMT (Pearson $r=0.23, p=0.04$ ) were associated with improvement while EZ disruption (Pearson $r=-0.24, p=0.05$ ) and COST disruption (Pearson $r=-0.32, p=0.01$ ) with deterioration in BCVA at 1 year. Table 1 depicts correlation between baseline demographics, OCT findings, and BCVA.

No significant differences were found between males and females $(0.08 \pm 0.39$ vs. $0.25 \pm 0.39$, respectively, $p=$ 0.08 , Student $t$ test) nor between patients with or without hyperreflective foci $(0.19 \pm 0.35$ vs. $0.19 \pm 0.49, p=0.99$, Student $t$ test). No significant differences were found in delta BCVA when examining the different vitreoretinal interface conditions ( $p=0.672$, analysis of variance). Categorical comparison of delta BCVA for BRVO patients is presented in Table 2. The partial least squares multiple regression analysis (Fig. 1) demonstrated that the factor most largely
Table 2. Categorical comparison of delta BCVA for BRVO patients

\begin{tabular}{lll}
\hline Parameter & Delta BCVA, logMAR & $p$ value \\
\hline Gender & & $0.08^{*}$ \\
$\quad$ Male $(n=25)$ & $0.08 \pm 0.39$ & \\
$\quad$ Female $(n=41)$ & $0.25 \pm 0.38$ & \\
Any intraretinal cysts & & $0.87^{*}$ \\
$\quad$ Yes $(n=60)$ & $0.22 \pm 0.40$ & \\
$\quad$ No $(n=6)$ & $0.19 \pm 0.41$ & $0.14^{*}$ \\
ERM & & \\
$\quad$ Yes $(n=5)$ & $-0.02 \pm 0.26$ & \\
$\quad$ No $(n=61)$ & $0.21 \pm 0.41$ & \\
Posterior hyaloid & & \\
$\quad$ ( $(n=30)$ & $0.16 \pm 0.49$ & \\
A $(n=28)$ & $0.22 \pm 0.33$ & $0.94^{*}$ \\
$\quad$ P $(n=8)$ & $0.19 \pm 0.33$ & \\
Hyperreflective dots & & \\
$\quad$ Yes $(n=42)$ & $0.19 \pm 0.35$ & \\
$\quad$ No $(n=24)$ & $0.19 \pm 0.49$ & \\
\hline
\end{tabular}

0 , complete attachment or posterior vitreous detachment; A, vitreomacular adhesion; $\mathrm{P}$, partial vitreous detachment. ERM, epiretinal membrane; BCVA, best-corrected visual acuity; BRVO, branch retinal vein occlusion. * Student $t$ test. ${ }^{* *}$ One-way analysis of variance was used for comparison of multiple group averages.

associated with improvement in BCVA was lower BCVA at baseline followed by greater CMT at baseline while the factor most largely associated with deterioration in BCVA was COST disruption followed by EZ disruption. 


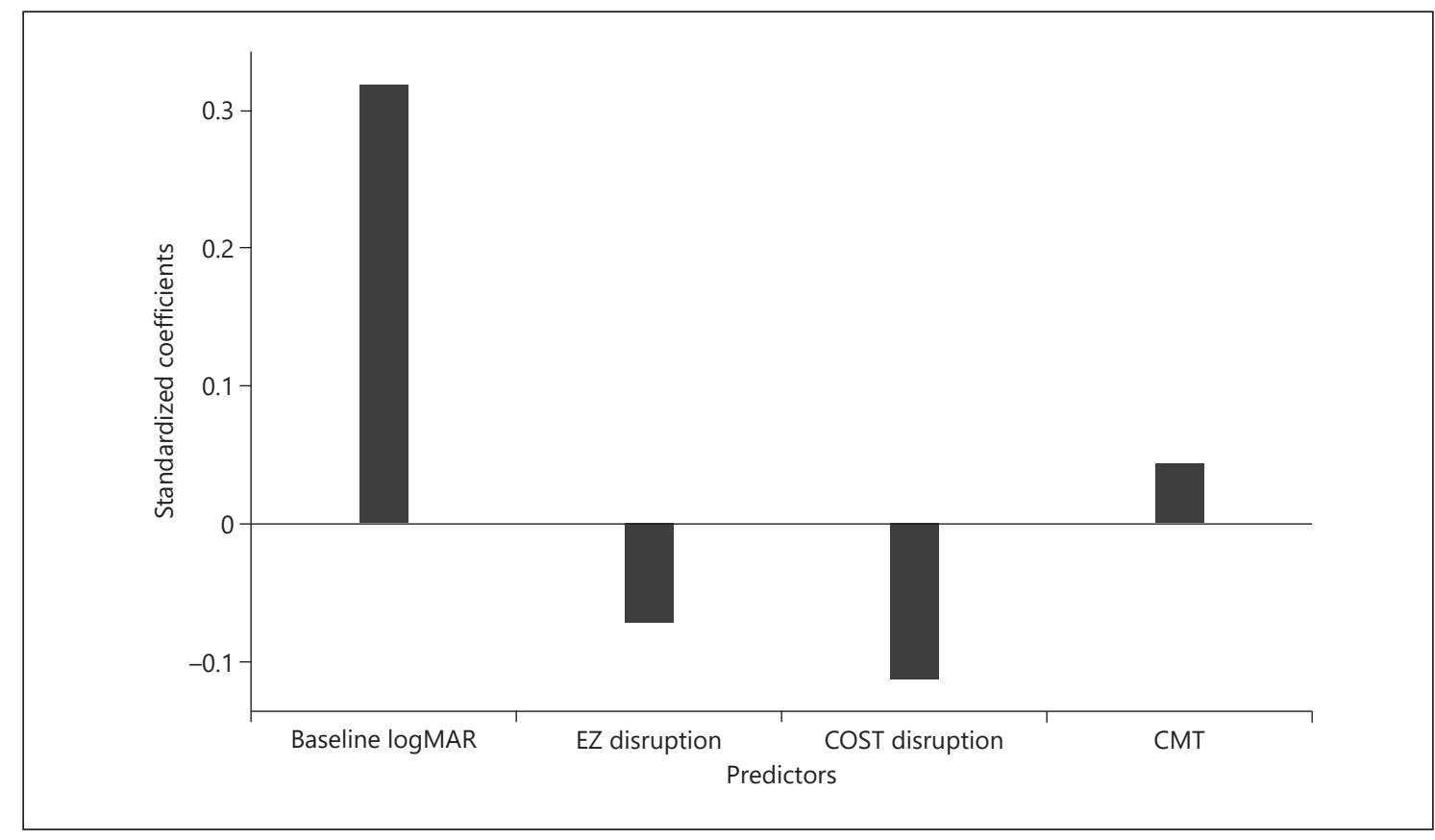

Fig. 1. Partial least squares multiple regression analysis of CMT, BCVA, COST disruption, and EZ disruption. CMT, central macular thickness; BCVA, best-corrected visual acuity; COST, cone outer segment tip; EZ, ellipsoid zone.

\section{Discussion}

The current study identified OCT parameters at baseline that may predict response to ME secondary to BRVO. Specifically, we identified CMT as well as extent of COST disruption and EZ disruption as significant markers. However, other factors including age of onset, epiretinal membrane, macular volume, SRF width or height, intraretinal cysts, DRIL, HF, microaneurysms, and ELM disruption were not significantly correlated with final VA. To the best of our knowledge, this is the first study to systematically characterize these aforementioned factors as potential SD-OCT biomarkers for ME secondary to BRVO.

Previous studies have tried to find predictive factors for successful BRVO therapy [19-22]. Several of them have focused on OCT findings and parameters. Most of them examined response to ranibizumab injections, and they provided conflicting results $[4,12,15,20,23]$. The conflicting results may be attributed to differences in methodology and patient population. However, few studies have evaluated the baseline SD-OCT characteristics of ME due to BRVO for visual outcome after intravitreal bevacizumab [12, 20, 22].

Kang et al. [20] have examined the SD-OCT patterns after intravitreal bevacizumab injection for BRVO. The factors Kang et al. [20] found to be significant predictors of final visual outcome were baseline BCVA and the sta- tus of photoreceptor IS/OS integrity in accordance with our study. The authors also found the status of the ELM to be correlated with the final visual outcome, which does not comply with our results. The possible reason for the difference could be different methodology, Kang et al. [20] measured discontinuity as present/absent and we measured quantitatively. Kang et al. [20] also examined baseline SRF as a predictor of final BCVA and found no correlation similar to our results.

Kang et al. [12] also found that the change in the number of hyperreflective foci correlated positively with change in BCVA but did not find correlation between the number of $\mathrm{HF}$ at baseline and final BCVA similar to our study. Opposed to our study, Kang et al. [12] did not find correlation between baseline macular thickness and final BCVA. The discordance could be related to different follow-up time, where Kang et al. [12] had only 6 months of follow-up [12].

Jaissle et al. [3] described 205 eyes of 204 patients with ME secondary to BRVO. Similar to our study, they found reduction in CMT, from a baseline of $454 \mu \mathrm{m}$ to $248 \mu \mathrm{m}$ after 48 weeks. Like Jaissle et al. [3], we also found significant correlation between baseline CMT and BCVA improvement. But, other previous studies have reported contradictory results, finding no significant correlation between baseline CMT and BCVA after intravitreal bevacizumab injection $[24,25]$. 
In this current study, we found a significant improvement in BCVA from 0.68 logMAR at baseline to $0.50 \log$ MAR at 1 year $(p<0.001)$. Jaissle et al. [3] also found similar improvement in BCVA, from 0.6 logMAR at baseline to $0.4 \log$ MAR at 48 weeks [4]. In contrast to our findings, previous prospective studies, such as BRAVO, VIBRANT, and BERVOLT [26], found BCVA improvement was 14-18 ETDRS letters. Explanations for the difference in BCVA improvement may be due to patient selection with a BCVA of 6/12-6/120, monthly follow-up, laser rescue, and more anti-vascular endothelial growth factor injections in the prospective trials [26]. In accordance with our study, Ota et al. [27] reported a significant correlation between initial total foveal thickness and final VA in BRVO patients with persistent ME documented by stratus OCT after treatment with grid laser photocoagulation, pars plana vitrectomy, or sub-Tenon injection of triamcinolone acetonide.

In the current study, SRF was not a significant predictor of improvement of BCVA at 1 year. Similarly, Hoeh et al. [19] examined CRVO and BRVO eyes assessed with time-domain OCT and reported that SRF was not a predictor of functional or anatomical outcomes following treatment with bevacizumab. They reported that final CMT and BCVA did not differ significantly between patients with or without baseline SRF [19].

Our group has previously reported change of DRIL following 3 monthly injections for CRVO and BRVO as a predictor of subsequent improvement following 1 year of treatment [22]. The current study did not assess change in SD-OCT parameters but rather focused on analysis of baseline parameters as predictors of change in BCVA at 1 year. Interestingly, there was no correlation between extent of DRIL at baseline and change in BCVA at 1 year. Taken together, this indicates that baseline DRIL extent does not predict change in BCVA at 1 year, while improvement in the extent of DRIL at 3 months (following 3 monthly injections) is a predictor of improvement in BCVA at 1 year.

This current study had several limitations: first, its retrospective design. Second, though we examined several OCT predictive factors, there is a possibility of other factors that may play a confounding role and have not been studied. Third, analysis of baseline OCT scans is difficult and might lead to misinterpretation because of the high edema. Looking at specific factors within the retina, including DRIL and COST, could be significantly impaired by the retinal thickness and the amount of intraretinal fluid, as well as intraretinal hemorrhages present in acute BRVO. Fourth, part of the baseline characteristics were missing, including time between occlusion and therapy, enlarged foveal avascular zone, and the presence of glaucoma. Last, in this current study, we examined the effect of bevacizumab injections only, whereas the effect of aflibercept or ranibizumab might be different. Future, larger, prospective studies, comparative between bevacizumab, ranibizumab, and aflibercept, may be considered.

\section{Conclusions}

The current study adds significant and new data to the few studies which have evaluated the baseline OCT characteristics of ME $[12,20,22]$ due to BRVO for visual outcome after intravitreal bevacizumab. Improvement in VA can be predicted based on predictive factors measured from the OCT. CMT, EZ disruption, and COST disruption were found as significant markers predicting VA improvement at 1 year.

\section{Statement of Ethics}

All data for this study were collected and analyzed in accordance with the policies and procedures of the Institutional Review Board (IRB) of the Meir Medical Center and the tenets set forth in the Declaration of Helsinki. Informed consent was not needed for this current study.

\section{Conflict of Interest Statement}

The authors have no conflicts of interest to declare.

\section{Funding Sources}

The authors have no funding sources to declare.

\section{Author Contributions}

Ori Segal contributed to design of the work; acquisition, analysis, and interpretation of data for the work; drafting the work; revising it critically for important intellectual content; final approval of the version to be published. Roy Yavnieli contributed to acquisition and analysis of data for the work; drafting the work; final approval of the version to be published. Michael Mimouni contributed to acquisition and analysis of data for the work; drafting the work; final approval of the version to be published. Gilad Rabina contributed to analysis and interpretation of data for the work; drafting the work and revising it critically for important intellectual content; final approval of the version to be published. Noa Geffen contributed to interpretation of data for the work; drafting the work and revising it critically for im- 
portant intellectual content; final approval of the version to be published. Elad Moisseiev contributed to design of the work; analysis and interpretation of data for the work; drafting the work and revising it critically for important intellectual content; final approval of the version to be published. Arie Y. Nemet contributed to design of the work; acquisition, analysis, and interpretation of data for the work; drafting the work and revising it critically for important intellectual content; final approval of the version to be published.

\section{Data Availability Statement}

All data generated or analyzed during this study are included in this article. Further enquiries can be directed to the corresponding author.

\section{References}

1 Rogers S, McIntosh RL, Cheung N, Lim L, Wang JJ, Mitchell P, et al. The prevalence of retinal vein occlusion: pooled data from population studies from the United States, Europe, Asia, and Australia. Ophthalmology. 2010 Feb;117(2):313-9.e1

2 Kolar P. Risk factors for central and branch retinal vein occlusion: a meta-analysis of published clinical data. J Ophthalmol. 2014;2014: 724780 .

3 Jaissle GB, Leitritz M, Gelisken F, Ziemssen F, Bartz-Schmidt KU, Szurman P. One-year results after intravitreal bevacizumab therapy for macular edema secondary to branch retinal vein occlusion. Graefes Arch Clin Exp Ophthalmol. 2009 Jan;247(1):27-33.

4 Jaissle GB, Szurman P, Feltgen N, Spitzer B, Pielen A, Rehak M, et al. Predictive factors for functional improvement after intravitreal bevacizumab therapy for macular edema due to branch retinal vein occlusion. Graefes Arch Clin Exp Ophthalmol. 2011 Feb;249(2):18392.

5 Rabena MD, Pieramici DJ, Castellarin AA, Nasir MA, Avery RL. Intravitreal bevacizum$\mathrm{ab}$ (Avastin) in the treatment of macular edema secondary to branch retinal vein occlusion. Retina. 2007;27(4):419-25.

6 Garnock-Jones KP. Ranibizumab: in macular oedema following retinal vein occlusion. Drugs. 2011 Mar 5;71(4):455-63.

7 Pielen A, Feltgen N, Isserstedt C, Callizo J, Junker B, Schmucker C. Efficacy and safety of intravitreal therapy in macular edema due to branch and central retinal vein occlusion: a systematic review. PLoS One. 2013 Oct 25; 8(10):e78538

8 Avitabile T, Longo A, Reibaldi A. Intravitreal triamcinolone compared with macular laser grid photocoagulation for the treatment of cystoid macular edema. Am J Ophthalmol. 2005 Oct;140(4):695-702.

9 Haller JA, Bandello F, Belfort R, Blumenkranz MS, Gillies M, Heier J, et al. Dexamethasone intravitreal implant in patients with macular edema related to branch or central retinal vein occlusion: twelve-month study results. Ophthalmology. 2011 Dec;118(12):2453-60.
10 Arnarsson Á, Stefánsson E. Laser treatment and the mechanism of edema reduction in branch retinal vein occlusion. Investig Ophthalmol Vis Sci. 2000 Mar;41(3):877-9.

11 Mandelcorn MS, Nrusimhadevara RK. Internal limiting membrane peeling for decompression of macular edema in retinal vein occlusion: a report of 14 cases. Retina. 2004 Jun; 24(3):348-55

12 Kang JW, Lee H, Chung H, Kim HC. Correlation between optical coherence tomographic hyperreflective foci and visual outcomes after intravitreal bevacizumab for macular edema in branch retinal vein occlusion. Graefes Arch Clin Exp Ophthalmol. 2014 Sep;252(9):141321.

13 Höh E, Schaal KB, Dithmar S. Central and branch retinal vein occlusion. Current strategies for treatment in Germany, Austria and Switzerland. Ophthalmologe. 2007 Apr; 104(4):290-4. German.

14 Kondo M, Kondo N, Ito Y, Kachi S, Kikuchi $M$, Yasuma TR, et al. Intravitreal injection of bevacizumab for macular edema secondary to branch retinal vein occlusion: results after 12 months and multiple regression analysis. Retina. 2009;29(9):1242-8.

15 Ach T, Hoeh AE, Schaal KB, Scheuerle AF, Dithmar S. Predictive factors for changes in macular edema in intravitreal bevacizumab therapy of retinal vein occlusion. Graefes Arch Clin Exp Ophthalmol. 2010;248(2):1559.

16 Segal O, Barayev E, Nemet AY, Geffen N, Vainer I, Mimouni M. prognostic value of hyperreflective foci in neovascular age-related macular degeneration treated with bevacizumab. Retina. 2016;36(11):2175-82.

17 Sun JK, Lin MM, Lammer J, Prager S, Sarangi $\mathrm{R}$, Silva PS, et al. Disorganization of the retinal inner layers as a predictor of visual acuity in eyes with center-involved diabetic macular edema. JAMA Ophthalmol. 2014;132(11): 1309-16.

18 Srinivasan VJ, Monson BK, Wojtkowski M, Bilonick RA, Gorczynska I, Chen R, et al. Characterization of outer retinal morphology with high-speed, ultrahigh-resolution optical coherence tomography. Invest Ophthalmol Vis Sci. 2008 Apr;49(4):1571-9.
19 Hoeh AE, Ach T, Schaal KB, Scheuerle AF, Dithmar S. Long-term follow-up of OCTguided bevacizumab treatment of macular edema due to retinal vein occlusion. Graefes Arch Clin Exp Ophthalmol. 2009;247(12): 1635-41.

20 Kang HM, Chung EJ, Kim YM, Koh HJ. Spectral-domain optical coherence tomography (SD-OCT) patterns and response to intravitreal bevacizumab therapy in macular edema associated with branch retinal vein occlusion. Graefes Arch Clin Exp Ophthalmol. 2013; 251(2):501-8.

21 Klein R, Klein BE, Moss SE, Meuer SM. The epidemiology of retinal vein occlusion: the Beaver Dam Eye Study. Trans Am Ophthalmol Soc. 2000;98:133-41; discussion 141-3.

22 Mimouni M, Segev O, Dori D, Geffen N, Flores V, Segal O. Disorganization of the retinal inner layers as a predictor of visual acuity in eyes with macular edema secondary to vein occlusion. Am J Ophthalmol. 2017;182:1607.

23 Narayanan R, Stewart M, Chhablani J, Panchal B, Pappuru R, Das T, et al. Baseline morphological characteristics as predictors of final visual acuity in patients with branch retinal vein occlusions: MARVEL report No. 3 . Indian J Ophthalmol. 2018;66(9):1291-4.

24 Kriechbaum K, Michels S, Prager F, Georgopoulos M, Funk M, Geitzenauer W, et al. Intravitreal avastin for macular oedema secondary to retinal vein occlusion: a prospective study. Br J Ophthalmol. 2008;92(4):518-22.

25 Hoeh AE, Ruppenstein M, Ach T, Dithmar S. OCT patterns of macular edema and response to bevacizumab therapy in retinal vein occlusion. Graefes Arch Clin Exp Ophthalmol. 2010;248(11):1567-72.

26 Shalchi Z, Mahroo O, Bunce C, Mitry D. Anti-vascular endothelial growth factor for macular oedema secondary to branch retinal vein occlusion. Cochrane Database Syst Rev. 2020 Jul;7(7):CD009510.

27 Ota M, Tsujikawa A, Murakami T, Yamaike N, Sakamoto A, Kotera Y, et al. Foveal photoreceptor layer in eyes with persistent cystoid macular edema associated with branch retinal vein occlusion. Am J Ophthalmol. 2008; 145(2):273-80. 